«Théâtralité, médialité et sociomédialité : Fondements et enjeux de l'intermédialité théâtrale »

\author{
Jean-Marc Larrue \\ Theatre Research in Canada / Recherches théâtrales an Canada, vol. 32, n² 2, 2011, p. 174-206.
}

Pour citer ce chapitre :

LARRUE, Jean-Marc, "Théâtralité, médialité et sociomédialité : Fondements et enjeux de l'intermédialité », Theatre Research in Canada / Recherches théâtrales au Canada, vol. 32, n² 2, 2011, p. 174-206. 


\section{JEAN-MARC LARRUE}

\section{THÉÂTRALITÉ, MÉDIALITÉ ET SOCIOMÉDIALITÉ : FONDEMENTS ET ENJEUX DE L'INTERMÉDIALITÉ THÉÂTRALE}

Née de l'avènement des technologies numériques, l'intermédialité recouvre des phénomènes aussi vieux que les plus vieux médias. Qu'on considère le théâtre comme un média ou qu'on lui reconnaisse des propriétés médiatiques, l'approche intermédiale renouvelle considérablement la compréhension que nous avons de sa nature et de sa conjoncture : ses rapports avec d'autres médias et d'autres pratiques, en particulier ceux et celles nés des technologies électriques puis numériques. La dynamique intermédiale repose autant sur le principe de remédiation tel que l'ont défini Bolter et Grusin que sur celui de reste et résidu-les médias résiduels d'Acland. Mais un média peut aussi bloquer la logique remédiante quand des agents considèrent que cette remédiation va à l'encontre des intérêts ou de l'identité du média. C’est ce que nous appelons la résistance médiatique dont l'histoire récente du théâtre offre de nombreux exemples. Parmi eux, celui de la très lente pénétration des technologies de reproduction sonore sur les scènes et dans les processus de création. Larticle suggère différentes hypothèses qui expliqueraient à la fois les causes, les modalités et les effets de cette résistance au théâtre au cours du Long Siècle (1880 à aujourd'hui), c'est-à-dire depuis la révolution électrique qui a vu, en même temps, naître la lampe à incandescence et le micro. Si la première a envahi toutes les scènes du théâtre occidental en moins de vingt ans, le second a été tenu à l'écart de la représentation pendant trois quarts de siècle.

Albeit the child of digital technology, intermediality is a phenomenon that embraces phenomena as old as the oldest cultural media. Regardless of whether theatre is recognized as a medium or just a discipline with mediatic properties, the intermedial perspective contributes much fresh insight to our understanding of theatre's nature and situation in terms of its relationship to other media and practices, particularly those generated by electricity or digital technology. The dynamics of intermediality reflect not only the process of "remediation," as defined by Bolter and Grusin, but also the kind of remains and residue represented by what Acland called "residual media." However, one medium can also block the remediating process when the people involved with the affected medium feel that such remediation harms their medium's interests or identity. This 
response is what we call "mediatic resistance" - and theatre's recent history is replete with examples of such reactions. One such example is the extremely slow adoption of sound reproduction technology on theatre stages and in the discipline's creative processes. This article offers various hypotheses concerning the causes, forms and effects of theatre's resistance to new media throughout the "Long Century" (1880 - present), i.e., since the electrical revolution successively brought us among other things incandescent lighting and microphones. While the former was universally adopted by Western theatre in the brief space of under 20 years, the latter was held at bay for some 75 !

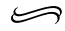

\section{Introduction}

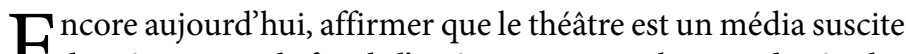
Cdes réactions et le fait de l'intégrer au vaste chantier des études intermédiales appelle des justifications. C'est cependant de cette double conviction-qu'il est un média et qu'il relève de l'intermédialité-que découle cet essai participant d'une réflexion en cours sur la lente et difficile pénétration du son électrique dans les scènes théatrales et sur la conceptualisation du phénomène de résistance médiatique qu'il a provoqué. Mais il n'y a pas d'essentialisme médiatique. Lorsqu'un média résiste à l'intrusion d'un autre média ou d'une nouvelle technologie-comme cela a été le cas avec le son électrique-, il n'agit pas comme un organisme vivant mobilisant des anticorps pour chasser ou détruire l'intrus. La résistance est toujours le fait du milieu-constitué d'hommes et de femmes-où opère le média. Ainsi, aux deux convictions de base évoquées à l'instant faut-il en ajouter une troisième : la destinée des médias et des technologies est intrinsèquement liée à leur milieu démergence ou d'appartenance.

Létude des socialités créées ou définies par les médias, la sociomédialité, est une composante primordiale de l'approche intermédiale. C'est pourquoi, tout en mettant au cœur de sa réflexion la matérialité ${ }^{1}$ sur laquelle repose nécessairement toute forme de médiation, l'intermédialité tient compte des rapports complexes et sans cesse à redéfinir entre les usagers et un média lui-même en perpétuel changement (provoqué par l'afflux incessant des nouvelles technologies, par les tentatives de pénétration des autres médias et des nouvelles technologies, par les pressions des usagers-leurs attentes, leurs besoins) ${ }^{2}$. Dissocier la tech- 
nique de l'usager en ignorant ce dernier reviendrait à une forme de technocentrisme et rouvrirait la voie à un déterminisme technologique que, justement, la recherche intermédiale s'est évertuée à battre en brèche. Le fait est à noter puisque on aurait pu s'attendre au contraire de la part d'une approche née dans le sillage d'une révolution technologique majeure, celle produite par l'avènement du numérique. Rappelons-le, l'intermédialité n’a pas vingt ans !

Le milieu joue donc un rôle nécessaire et déterminant dans l'avènement ${ }^{3}$ - ou le non-avènement-des médias. Cette affirmation, en apparence anodine, a des incidences heuristiques considérables. Le milieu et les médias ne cessent de se co-transformer, de se co-construire et de se co-détruire. Disons-le autrement : les médias naissent du milieu qu'ils contribuent à former. C'est d'ailleurs pour souligner cette indissociabilité des médias et des socialités qui leur sont associées qu’a été développé cet axe sociomédial que je viens dévoquer et dont il est question dans la dernière partie de ce texte.

\section{L'intermédialité et la nouvelle litéracie}

L'intermédialité est tout le contraire d'un système fermé et c'est à tort qu'on la réduit à un simple concept. Polysémique, multidimensionnelle et mouvante, l'intermédialité transcende les frontières disciplinaires. À l'instar de Jürgen Müller, l'un des pionniers allemands du domaine, nous dirions delle que c'est un axe de pertinence, axe qui a la particularité de mettre en lien des systèmes dynamiques plutôt que des objets aux propriétés et contours définis. Dans un des textes fondateurs de l'intermédialité parus en français, Éric Méchoulan inscrit l'intermédialité dans un mouvement dont elle serait le troisième temps-après l'intertextualité et l'interdiscursivité-et qui affirme la primauté de la relation.

[L]a relation est par principe première [...]. Là où la pensée classique voit généralement des objets isolés quelle met ensuite en relation, la pensée contemporaine insiste sur le fait que les objets sont avant tout des nœuds de relations, des mouvements de relation assez ralentis pour paraître immobiles. (Méchoulan 11)

Mais la formidable accélération provoquée par les technologies numériques ne laisse même plus d'illusion d'immobilité : tout est mouvement et en mouvement. Sans doute, la principale « illusion perdue » quévoque Méchoulan ${ }^{4}$ est que l'immobilité n’est- 
nétait-qu'apparence : elle n'existe pas. On peut ainsi assimiler la naissance de la pensée intermédiale à cette prise de conscience brutale : tout est processuel. Il s'agit là d'un changement radical non seulement dans lapproche scientifique des phénomènes de communication mais dans l'organisation des savoirs. Tenant l'intermédialité à la fois comme symptôme et conséquence de ce changement paradigmatique majeur, Silvestra Mariniello voit en l'intermédialité une autre manifestation de la fin du langage comme médiateur universel et unique.

En d'autres termes, notre litéracie en est encore une qui nous confine dans une configuration du savoir centrée sur le langage (logos) et ne nous donne pas les moyens de comprendre notre vie avec les nouveaux médias (outre la photographie et le cinéma, les médias électriques et électroniques), sauf à partir de la perspective du logocentrisme. (Mariniello 166)

Récusant cette litéracie logocentrique qui est un produit du langage et qui, comme ce dernier, est fondée sur l'abstraction et sur le système des oppositions, Mariniello relève des manifestations marquantes - ni isolées ni prodromiques-de l'instauration d'une autre litéracie qui ne reposerait pas sur des entités stables, duelles et discrètes, organisées selon un système d'opposition binaire sans lien avec la réalité (dont le langage l'a abstraite), mais sur le principe de la différence. En ce sens, la réflexion de Mariniello est à rapprocher des travaux de la critique déconstructiviste de Yale et, en particulier, de ceux de Barbara Johnson (1980).

À la fin de l'illusion de l'immobilité, qui entraîne la fin du règne de l'objet aux limites nettes, s'ajoute le rejet des dualismes structurants. Il faut insister sur cet aspect: si l'intermédialité ne concernait-de nombreux chercheurs la conçoivent encore ainsi-que létude des rapports entre les médias et si elle ne faisait que reprendre les approches et acceptions traditionnelles des médias et des arts, elle ne ferait que préciser et fragmenter ce qui existe déjà-l'écologie des médias, la médiologie, etc.—et ne serait qu'une autre façon de dire l'interdisciplinarité. Mais l'intermédialité est d'un autre ordre. Elle bouleverse nos conceptions de la médiation et des médias. Laction des technologies numériques n’y est évidemment pas étrangère. Leur dynamique invasive (elles s'insinuent partout), leur formidable capacité de migrer d'un environnement à l'autre en préservant leur intégrité, leur effet de contamination et d'hybridation font vaciller les modèles établis. 
On ne peut plus envisager létude des phénomènes médiatiques et de communication à partir de catégories sûres et stables, des catégories dont les contours paraissent désormais si flous et si poreux qu'elles perdent toute spécificité transcendante, toute potentialité opératoire.

Et on comprend, vu la complexité des enjeux soulevés et la vastitude des espaces à couvrir, le refus de lépistémologie intermédiale de réduire l'ensemble de ces phénomènes et de ces champs à un concept, si puissant soit-il ! Lintermédialité n’est pas un concept mais un axe de pertinence qui concerne « des processus de processus » et une dynamique du croisement, dynamique qui peut être tour à tour ou simultanément favorisée, accélérée, suspendue ou rejetée. Quand le croisement est possible, il s'effectue selon diverses modalités qui peuvent aller jusquà la symbiose-et les innombrables variantes du commensalisme et du mutualisme. Le média hôte, dans ce cas, peut donc subir l'intrus ou en tirer profit, parfois les deux simultanément ou en alternance, les deux médias symbiotes pouvant être tour à tour ou en même temps prédateurs, parasites, esclaves, partenaires, avec tous les rapports de pouvoir et d'influence que cela suppose.

Mais le processus peut avorter, être bloqué dès le départ ou en cours de route. Jusquà présent, les croisements fusionnels aboutis, qui résultent en la formation de nouveaux médias, ont davantage attiré l'attention des chercheurs que les cas d'inaboutissement dus au rejet et au blocage. On peut comprendre pourquoi : ces derniers sont plus difficiles à déceler et à cerner, moins spectaculaires, moins séduisants et leur valeur exemplaire reste limitée. On présume aussi qu'ils peuvent moins contribuer à la modélisation d'une nouvelle généalogie des médias. Les choses ne sont cependant pas aussi nettes et nous nous rendons compte qu'il y autant à tirer de ces essais inaboutis que des réussites, que ces réussites elles-mêmes portent inévitablement une part d'échec, en résultent.

Cet élargissement de la perspective de létude des médias et le recul épistémique et historique qu'il impose ont permis une première ébauche de définition ${ }^{5}$ de l'intermédialité quon pourrait articuler ainsi : l'intermédialité est un axe de pertinence qui met en lien dans un mouvement dynamique, à la fois continuel, multidirectionnel et non linéaire :

(A) les relations entre les médias ;

(B) le creuset de médias et de technologies d'où émerge et s'institutionnalise peu à peu un média particulier; 
(C) les socialités complexes résultant de lévolution des médias et y participant ${ }^{6}$

(D) la matérialité inhérente et centrale à toute opération de transmission, de représentation et d'archivage de l'expérience;

(E) la nouvelle litéracie (que, en toute logique, on pourrait nommer « médiacie»).

Contrairement à ce que les circonstances originelles de l'intermédialité auraient pu suggérer-le choc des nouveaux médias (numériques)—, l'intermédialité est transhistorique. Elle recouvre en effet des phénomènes qui ne sont pas propres à cette «third wave of technologizing of the world, from orality to literacy to mediacy (media literacy or secondary orality)" (Blakesley). L'intermédialité existait effectivement bien avant les nouvelles technologies. Certains chercheurs (Moser, Boenisch) la font remonter jusquà la basse antiquité (la naissance de l'alphabet) et les travaux sur l'avènement de cette autre révolution technologique qu'a été l'électricité 7 montrent bien, par les similitudes frappantes qu'on observe dans la formation des médias électriques et des médias numériques, que les mêmes phénomènes sont souvent à l’ouvre ${ }^{8}$ à cent ans décart.

Rappelons que c'est grâce à leur remarquable puissance transformationnelle et à la rapidité de leur action que les nouvelles technologies ont rendu tout cela immédiatement perceptible, ${ }^{9}$ permettant de voir des mouvements qui, il n'y a pas si longtemps, passaient inaperçus ou, pour reprendre les termes de Méchoulan, passaient pour de l'immobilité. En fait, les technologies numériques ont servi de révélateur à une dynamique intermédiale aussi ancienne que les médias eux-mêmes. Et ce nest évidemment pas un hasard si ces bouleversements ${ }^{10}$ débutent à peu près au moment où les études théâtrales migrent à l'extérieur du champ des études littéraires où elles se sentent trop à létroit parce que, justement, la théâtralité et la performativité n'y sont pas suffisamment prises en compte. Cette migration, qui a résulté dans la création d'innombrables départements et programmes d'études théâtrales (théoriques et pratiques) depuis les années 1980 correspond bien à ce mouvement plus vaste évoqué par Mariniello-le passage d'une litéracie logocentrique à une litéracie de la différence-et que James Cisneros analyse du point de vue des institutions. Constituée sur un modèle de savoir disciplinaire fondé sur la pensée des Lumières, l'université vit sans doute l'une des pires crises de son histoire parce quelle ne parvient pas à transcender les barrières disciplinaires et à passer 
véritablement à l'ère de l' "inter » qu'elle préconise pourtant et dont procède l'intermédialité ${ }^{11}$.

\section{Média, théâtre et technologie}

Cette nouvelle approche des phénomènes de communication et cette reconfiguration des savoirs ne font pas table rase de tous les acquis. Elles nont pas modifié notre compréhension de la fonctionnalité des médias telle que lont définie, en 1996, Frédéric Barbier et Catherine Bertho Lavenir, pour lesquels un média est :

tout système de communication permettant à une société de remplir tout ou une partie des trois fonctions essentielles de la conservation, de la communication à distance des messages et des savoirs, et de la réactualisation des pratiques culturelles et politiques. (Barbier et Lavenir 5)

Cette définition ne mentionne ni la dimension matérielle de la médiation ni la fonction des usagers. Celle que propose Éliséo Véron en tient compte :

Un média, c'est : [...] un ensemble constitué par une technologie PLUS [souligné par l'auteur] les pratiques sociales de production et d'appropriation de cette technologie, lorsqu'il y a accès public (quelles que soient les conditions de cet accès, qui est généralement payant) aux messages. (Véron 51)

La définition fonctionnaliste et très générale de Barbier et Lavenir- " tout ou une partie " - ne provoque évidemment aucune résistance chez les théoriciens du théâtre, mais la définition structurelle de Véron peut causer problème quand on s'en tient à une acception étroite du terme « technologie». En effet, si on exclut les spectacles à forte composante technologique tels ceux du Wooster Group, de Robert Lepage, de Guy Cassiers, de Nicolas Stemann ou du Big Art Group, ${ }^{12}$ pour ne citer queux-où la technologie est présente à toutes les phases de la production et de la représentation et y joue un rôle déterminant-, si on admet sans difficulté que la scène à l'italienne-et sa machinerie illusionniste-aussi bien que les systèmes actuels de sonorisation et déclairage des théâtres sont des technologies hautement sophistiquées, on conçoit mal que lacteur jouant sur scène relève du paradigme technologique, que sa voix, son geste, son souffle soient des composantes d'une quelconque technologie théâtrale.

Cette résistance n'est pas nouvelle. Peter Boenisch rappelle que le mouvement vers le minimalisme, voire l'essentialisme théâ- 
tral-la scène vide-qu'incarnent Jerzy Grotowski et Peter Brook serait justement une réaction à l'envahissement de l'environnement de l'époque par les technologies. Le théâtre se serait ainsi défini comme un art de l'essentiel et le lieu théâtral comme un abri antitechnologique! La création collective, qui bouleverse les pratiques dominantes entre 1968 et 1976, va dans le même sens, celui d'une quête d'authenticité dans la relation scène-salle dont, du reste, elle abolit les barrières (Baldwin, Larrue et Page) ${ }^{13}$. L'authenticité est alors perçue comme l'absence de toute médiation, de toute technologie. "In its simplest form", rappelle Chiel Kattenbelt, "theatre can exist without any technology " (Kattenbelt 37).

Le terme " technologie » heurte la sensibilité théâtrale actuelle, mais il n'est pas aisé de lui trouver un substitut satisfaisant. C'est dire la complexité des phénomènes en jeu ici, ceux de la médiation comme ceux de la réalité qui en est l'objet. Le concept de "dispositif » serait sans doute plus acceptable. Lié à l'idée du "tournant optique " -pictorial turn-défendue par W.J.T. Mitchell (11-35), ${ }^{14}$ il a été largement repris par les spécialistes du cinéma des premiers temps et comporte désormais une connotation cinématographique. Ce n'est plus un concept tout à fait neutre, contrairement à celui d' " appareil ${ }^{15}$ " que Jean-Louis Déotte a élaboré à partir de la logique lyotardienne des inscriptions. Si l'inscription, dans son principe, répond au besoin vital et continu de transmettre et de pérenniser l'information, les "surfaces » sur lesquelles elle se fait changent selon les époques. ${ }^{16}$ Et Lyotard centre son attention sur « la nature du lieu d'inscription " puisque c'est bien là-dans cette matérialité-que se trouvent en bonne partie les clés de la médiation.

Le concept d'appareil développé à partir de là par Déotte et ses collaborateurs se distingue de celui de dispositif, de prothèse ou de «médium » par sa dimension immatérielle-qui s'ajoute à la matérialité de l'inscription—et par son lien avec le milieu.

Par appareil, il faut entendre ce qui fait époque dans l'accueil de l'événement, par définition imprévisible. Il s'agit d'un certain rapport-technique-que le corps entretient avec la loi, selon des régimes spécifiques, et non d'un simple prolongement d'une fonction corporelle hors du corps lui-même. (Froger 6 note 8 )

Cette première clarification établie, Déotte aborde la question du processus et de son effet que Michèle Garneau synthétise de la façon suivante : 
Le cinéma, comme la perspective, la camera obscura, le musée, la photographie, la cure analytique, est un appareil de la modernité. [...]. Ce qui définit un appareil, c'est son infrastructure projective (l'appareil est un producteur d'images), ainsi que son potentiel émancipatoire (contrairement au « dispositif » qui, lui, est du côté du pouvoir). Hypothèse à considérer : les appareils entraîneraient des changements peut-être plus importants que les révolutions politiques. Si l'on veut comprendre l'histoire, ou encore, la sensibilité d'une époque, —sensibilité qui, selon Déotte, est toujours « appareillée » -, il faut se pencher sur ses appareils. (Garneau 75 note 118)

Le concept d'appareil a l'avantage de transcender la technique tout en l'englobant. Dans certains cas, d'où son intérêt pour le théâtre, il peut recouvrir une dimension esthétique, l'esthétique étant, selon Déotte, "le faire-monde de certains appareils, lesquels génèrent une sensibilité commune, donc l'esthétique au sens large d'aisthésis, celle qui génère aussi des valeurs, des manières collectives d'être et de dire : un ethos et aussi finalement un art » (Déotte 264).

Mais il faut reconnaitre avec Johanne Villeneuve que l'appareil-comme le "dispositif " - peine à rendre compte de la complexité de certaines médiations et reste trop fortement modélisé par la perspective ocularocentriste-de la vision-dont il est issu. Le corps agissant et parlant de l'acteur sur scène ne peut pas être assimilable à l'inscription-le fait d'inscrire et ce qui est inscrit-sur le corps primitif. La peinture, la photographie, l'exposition, le cinéma supposent tous une opération de mise à distance-l'inscription sur une surface-que, même métaphoriquement, on ne peut retrouver telle quelle au théâtre ni dans les arts du spectacle vivant. Il y a un autre problème.

La théorie des appareils, souligne Villeneuve, [tient] pour acquis le modèle scripturaire, celui de l'écriture et de l'inscription, ce qui rend délicate aux extrêmes toute appréhension de l'oralité dans la complexité de ses manifestations propres et de ses époques. (Villeneuve 209)

Or, l'oralité et, plus globalement, le son sont aussi importants que l'image dans la tradition théâtrale, bien que l'histoire du théâtre reste très imprécise à ce sujet ${ }^{17}$. La théorie des appareils nécessite donc d'être approfondie et d'être plus rigoureusement éprouvée, mais son potentiel heuristique est indiscutable et le concept 
d'« appareil » offre l'avantage de susciter moins de résistance, dans le monde du théâtre, que celui de technologie ou celui de dispositif (encore trop associé au cinéma).

\section{Théâtralité, médialité et remédiation}

Toutes ces questions, qui tiennent autant de l'ontologie que de lépistémologie théâtrale et qui concernent aussi bien la période actuelle que le théâtre passé, nous ramènent à un autre débat de fond, celui qui touche à la spécificité du théâtre. Cette spécificité ne peut évidemment pas, dans une perspective intermédiale, être construite sur le mode binaire des oppositions entre ce qui est du théâtre, ce qui ne l'est pas ; elle n'est pas non plus figée-immobile-, bien que certaines des caractéristiques de la pratique théâtrale aient traversé les époques et jouissent d'une certaine pérennité, telles la "coprésence " de l'acteur et du spectateur. Notons aussi que rien n'interdit que ces caractéristiques soient empruntées par ou à d’autres pratiques ou médias. Lère n'est plus à l'exclusivité.

Formulons la chose autrement : ce qui caractérise le théâtre est un ensemble instable de normes, de processus et d'objets qui ne lui sont pas nécessairement propres. Et c'est pourtant de cet ensemble indéterminé, mouvant, en continuelle interaction et transformation, que se dégage la théâtralité. La théâtralité ne serait-elle qu'affaire de conjoncture ? C'est ce que suggère Willmar Sauter: « [theatricality] can only be defined within a certain time and a certain culture " (Sauter 52). Thomas Postlewait insiste, quant à lui, sur la dimension performative nécessairement attachée à la " coprésence »: la théâtralité serait « a way of describing what performers and what spectators do together in the making of 'the theatrical event' » (Sauter np, qtd. Davis et Postlewait 23). Mais que vaut cette définition, fondée sur la coprésence, pour une pratique que la virtualisation et le différé envahissent un peu plus chaque jour?

D’un point de vue intermédial, la théâtralité n'est rien d'autre que la médialité du théâtre, ce qui le singularise en tant que média. Et cette médialité résulte des modes ${ }^{18}$ de médiation particuliers (Elleström) qu'utilise le théâtre pour produire ses spectacles. Reprenant la formule choc de Jay David Bolter et Richard Grusin- " $[\mathrm{A}]$ medium is that which remediates " (Bolter et Grusin 65), qui pourrait bien être l'un des slogans-clés de l'intermédialité-, Peter Boenisch présente la médialité comme la remédiation : "mediality as remediation " (Boenisch 107). Cette équation en apparence simple appelle quelques explications. 
Évoquant l'idée de Marshall Mcluhan selon laquelle tout média porte en lui dautres médias potentiels en même temps que les traces de médias antérieurs, Bolter et Grusin ouvrent différentes pistes-toujours liées à des processus, non à des états, non à des objets—qui ne sont pas sans intérêt pour le théâtre :

[A medium] appropriates the techniques, forms and social significance of other media and attempts to rival or refashion them in the name of the real. A medium in our culture can never operate in isolation, because it must enter into relationships of respect and rivalry with other media. (Bolter et Grusin 65)

Ces emprunts incessants de techniques, de formes, de sens social, justifiés par le besoin proclamé de toujours mieux représenterou produire-le réel, expliquent la nature et la dynamique des médias qui sont le produit d'hybridation ou qui sont eux-mêmes des réseaux- « networks or hybrids » (Bolter et Grusin 19). Dans la perspective théâtrale, l'adéquation de Peter Boenisch-médialité / remédiation-est d'autant plus justifiée que le théâtre est largement compris comme un regroupement de médias, un média composite, un média de médias ou un " hypermédia " (Kattenbelt 29-40). Le principe de remédiation-même si le terme n'est pas toujours présent-est à la base d'une multitude de recherches intermédiales qui, sans toujours porter directement sur le théâtre, en changent inévitablement notre perception. Qu'est-ce qui, précisément, est remédié, quest-ce qui remédie? Et comment ? Il y a évidemment des enjeux ontologiques majeurs à la remédiation et ce nest pas un hasard si l'un des ouvrages les plus discutés dans le milieu anglo-saxon ${ }^{19}$ de la recherche théâtrale au cours de ces dernières années, Liveness : Performance in a Mediatized Culture de Philip Auslander (publié une première fois en 1999), a justement porté sur la notion de présence.

La définition classique du théâtre sest en effet réduite comme peau de chagrin, il n'en reste plus, comme nécessité ultime ou dernier rempart, que la " coprésence " (de l'acteur et du spectateur). Or ce dernier verrou (qu'il partage avec les autres arts du spectacle "vivant» ou " arts de la présence ») risque de sauter sous l'effet des nouveaux médias-l'acteur peut être présent en temps réel mais non dans l'espace, il peut être présent dans lespace mais occulté par sa propre image médiatisée, il peut donner l'illusion dềtre là mais ne l'est pas, être fragmenté, etc. Les risques sont tels que les chercheurs parlent plus prudemment-et justementaujourd'hui d'«effets de présence ».

184 • TRiC / RTaC • 322 (2011) • Jean-Marc Larrue •pp 174-206 
Au-delà de la question de la présence et du direct, ce que l'ouvrage d'Auslander démontre avec force c'est que les médias qui effectuent cette remédiation perpétuelle en sont aussi le produit. Que le cinéma des premiers temps ait largement emprunté au théâtre-ses stratégies narratives, sa frontalité, ses agents-tient aujourd'hui de l'évidence, mais que la télévision, fondée sur la technologie de la caméra et du film, ait davantage remédié le théâtre que le cinéma, voilà qui étonne et complique les choses. Se distinguant du cinéma, qui assume pleinement son effet médiatisant (on sait qu'on ne voit qu'une représentation des acteurs, l'acteur étant remédié en signal lumineux projeté- « inscrit »-sur une surface plane, l'écran, dont on ne peut faire abstraction), la télévision a emprunté au théâtre l'effet d'immédiateté et de coprésence qui le caractérise : c'est l'invention du " direct » - le live-, une coprésence médiatisée mais, en principe, en temps réel, qui n'est d'ailleurs souvent qu'un simulacre de coprésence (quand il y a pré-enregistrement). Ces appropriations ne sont évidemment ni définitives, ni limitées, ${ }^{20}$ ni à sens unique.

Les travaux qu'André Gaudreault et Philippe Marion ont menés parallèlement à Auslander sur la généalogie du cinéma identifient les conditions de la remédiation et quelques momentsclés du processus dans la constitution du média cinématographique. Là encore, il faut comprendre que la dynamique transformationnelle n'est ni inéluctable ni unidirectionnelle, ${ }^{21}$ que des retournements, des rejets, des interruptions, des blocages, sont toujours possibles. Mais si la remédiation réussit, il en résulte nécessairement un nouveau média. Le modèle de la « double naissance » que Gaudreault et Marion dégagent de leurs observations du cinéma des premiers temps correspond à un processus en trois temps marqués par l'apparition d'une nouvelle technologie (phase 1), par la mise en place d'un dispositif et d'une nouvelle pratique (phase 2) et par la prise de conscience et l'affirmation d'une autonomie expressive (qui correspond à l'opacité-hypermediacy de Bolter et Grusin) et d'une spécificité médiatique avec tout ce que cela suppose, tant pour les socialités que pour la dimension institutionnelle du média (phase 3). François Odin observe le même mouvement qualifiant la technologie émergente de «cryptomédia » (puisqu'on ne peut pas encore prévoir qu'elle va donner naissance à un média), la phase 2 marquerait l'apparition du " protomédia ", et la phase 3 , celle du média proprement dit (Gaudreault et Marion 11-36).

$\mathrm{Ni}$ les technologies ni les pratiques médiatiques (celles des usagers) n'apparaissent ex nihilo. Avant le cinématographe, il y a eu la lanterne magique, la peinture, la photographie, les progrès 
de lélectricité-l'invention de la lampe à incandescence, etc. Toute nouvelle technologie-et plus globalement toute pratique médiatique ou artistique-s'inscrit nécessairement dans des " séries culturelles » préexistantes qu'il faut combiner au concept d' " appareil " de Déotte et qui remettent à l'avant la question essentielle des socialités dans la dynamique intermédiale. Cette dernière question reste pourtant largement ignorée de la théorie.

Même dans les histoires / archéologies innovatrices récentes [rappelle Jürgen Müller], l'aspect social des processus intermédiatiques n'est pas abordé. Notre démarche, qui essaie de ne pas répéter les manques des recherches précédentes, consiste donc à nous préoccuper non seulement des aspects médiatiques, technologiques, génériques des rencontres des médias et de leurs productions, mais aussi et tout particulièrement à nous pencher sur les fonctions nées de ces rencontres qui déploient leur processus dans des réseaux de séries culturelles. Selon notre approche, les "fonctions " de ces processus sont à comprendre comme un éventail d'effets possibles ou / et réalisés par les producteurs et-avant tout-pour les «utilisateurs» ou les récepteurs des médias et de leurs productions, que ce récepteur soit un individu ou un groupe social spécifique. (Müller 95)

La série culturelle est elle-même le produit de ces « rencontres » et les socialités participent pleinement à l'échange : "Les effets peuvent se produire à différents niveaux : au niveau de certaines réactions / activités cognitives / émotionnelles et esthétiques, comme au niveau de notre comportement social » (Müller 95). Si le cinéma sest progressivement défini à partir de diverses séries culturelles, comme tout média ou pratique artistique, il en emprunte aussi les habitus et l'ethos des usagers-habitués-récepteurs-producteurs. Si lélectricité, la photographie, etc. ont joué un rôle déterminant dans le développement du Cinématographe (le dispositif) puis du cinéma (la pratique médiatique), la coprésence des spectateurs (voir-ensemble) et la frontalité (être face au spectacle), qui étaient des caractéristiques comportementales du spectateur de théâtre, ont été transmises au spectateur de cinéma et adoptées par lui.

\section{L'interartialité : la remédiation dans les médias artistiques}

Les travaux d'Auslander, Müller, Gaudreault et Marion traitent de la remédiation dans une perspective généalogique, donc nécessai- 
rement linéaire. Ceux de Klaus Clüver et Walter Moser (Moser 69-92), spécialistes des transferts culturels, portent sur des phénomènes synchrones et, de ce fait, élargissent sensiblement la portée du concept de remédiation qu'ils observent lorsque deux formes d'art-donc deux médialités-se rencontrent au cours du processus créatif. C'est le cas, par exemple, quand il y a adaptation ou quand une pratique artistique traite d'une autre pratique artistique (le cinéma de la peinture). Se pose alors le défi du passage de la coexistence initiale de deux médialités à la prise en charge de l'une par l'autre. Les cas analysés par Moser concernent les rapports entre poésie et peinture, poésie et musique, peinture et cinéma, dans trois époques et conjonctures différentes.

Soulignant que ces rapports sont aussi vieux que les arts euxmêmes, Moser recourt au concept d'interartialité, qu'il faut comprendre comme une modalité spécifique de l'intermédialité, ${ }^{22}$ modalité qui présente l'immense avantage de prendre en compte le changement de statut et de définition du réel selon les pratiques et les époques. Reprenant à son compte la double métaphore de la lampe et du miroir créée par Meyer Abrams pour caractériser la révolution romantique-le passage de la mimèsis à la poïesis-, Moser observe que, avec le romantisme, «[1]e modèle artistique nest plus un art qui imite et qui copie, mais un art nonreprésentatif dont on rend compte non pas en termes de vérité et d'adéquation à un objet à représenter, mais en termes dénergie émotive " (Moser 74). La poésie, qui était jusque-là l’art dominant, le "modèle ", cède la place à la musique, art du son et de la performance du corps pour lequel le réel est ce quon voit et entend dans le hic et nunc (nous sommes avant l'époque des technologies de reproduction sonore). Que le site de la réalité soit dans ce que l’ouvre représente ou dans l’ouvre elle-même ${ }^{23}$ ne change rien à la capacité de l'interartialité de rendre compte du fonctionnement de la remédiation et des stratégies qui y conduisent et qui la gouvernent.

Non seulement l'interartialité confirme-t-elle que le modèle de Bolter et Grusin s'applique aux arts-ce qui fortifie l'hypothèse que les arts sont des médias-, elle démontre aussi comment et en quelles circonstances se déclenche le principe dynamique par lequel tout média oscille de la transparence (qui provoque l'immédiacie) à l’opacité (liée à l'hypermédiacie) :

[L]a médialité d'un art qui tend à s'auto-effacer dans un semblant de transparence, devient nécessairement apparente quand deux médias différents entrent en jeu et interfèrent [...]. 
Le média apparaît alors, prenant la consistance d'une opacité. Il émerge donc, en tant qu'objet de connaissance d'une relation intermédiale qui l’aurait (toujours déjà) précédé. (Moser 80 )

Ces travaux sont d'une grande résonance pour le théâtre. D’abord, parce qu'ils portent spécifiquement sur le monde des arts, sur la nature de l'œuvre artistique et sur la question du réel. Ensuite, parce qu'ils démontrent que la remédiation ne se limite pas à une logique de lutte de pouvoir et de lutte pour la survie entre les médias, mais qu'elle est présente au cœur même de tout processus créateur. On regrette que Moser ne se soit pas risqué plus avant sur le terrain de lontologie artistique et nait pas abordé la question de lart actuel où, comme dans les médias, les hiérarchies et les frontières se brouillent.

Nous supposons donc que si la fonction de lart est avant tout de créer des ouvres d'art, l'interartialité devrait être le modus operandi dominant des pratiques artistiques contemporaines-qui se fondent sur les transferts et les hybridations. L'interartialité revêt une importance plus grande encore si, délaissant les définitions de nature procédurale ou fonctionnelle de l'art, on admet que l’euvre ne peut acquérir son statut artistique, donc ne peut se qualifier comme œuvre d'art, que par sa dimension d'historicité intentionnelle. Les théoriciens de l'intentionnalité en art, Jerrold Levinson, Noël Carroll et, au moins partiellement, Arthur Danto ${ }^{24}$ considèrent en effet que, faute d'archétypes indiscutables, de critères fonctionnels ou esthétiques sûrs, de mode de production stables, on ne peut définir l'art, lui donner un contenu ontologique autrement que par un système de relation intentionnelle entre le projet artistique en cours et ce que l'art a été. En d'autres termes, cest l'inscription de l'œuvre dans l'histoire de l'art qui confère à cette œuvre sa valeur artistique. Et pour que l'œuvre s'inscrive dans cette histoire, il faut que le créateur-ou son ayant droit-ait l'intention de l'inscrire dans cette histoire. Dans cette perspective, l'interartialité apparaît comme une nécessité dans la mesure où l'oeuvre produite par la remédiation l'est de façon intentionnelle et découle nécessairement de l'art passé : elle participe de ce fait à l'histoire de l'art. Cet aspect de l'interartialité est évidemment à lier aux stratégies démergence des nouveaux médias et de leur inscription dans des séries culturelles qui leur préexistent. L'intentionnalité n'est pas propre à l'interartialité. Elle souligne cependant la fonction centrale des agents dans tout phénomène de remédiation et rappelle la nécessaire prise en compte des socialités-donc de la sociomédialité-dans les processus intermédiaux. 
Tout en relevant de l'intermédialité, l'interartialité en révèle ainsi des aspects importants et jusque-là peu étudiés. Son apport est considérable. D’abord, elle situe la remédiation sur un plan synchronique-entre des pratiques actives contemporainesplutôt que dans une perspective de successivité ; ensuite elle montre que les rapports traditionnels de pouvoir-dominant et dominé, intégration, subordination-ne peuvent s'appliquer tels quels aux pratiques artistiques et, plus largement, médiatiques (puisque le média hôte peut aussi bien s'effacer que sopacifier en présence du média intrus ${ }^{25}$ ). Moser n'a malheureusement pas approfondi cet aspect du sujet et n'a pas non plus étendu son étude au théâtre. C'est regrettable puisque tout porte à croire que, au sein de ces médias particuliers que sont les arts, le théâtre est atypique. Son mode de remédiation singulier n'est sans doute pas unique mais rien n'indique qu'il soit habituel. ${ }^{26}$

\section{L’ " hypermédia théâtre " et l'intermédialité}

C'est à l'étude de certains aspects de cette singularité que Peter Boenisch et Chiel Kattenbelt se sont consacrés dans des articles parus dans l'ouvrage collectif Intermediality in Theatre and Performance publié en 2006 sous l'égide de la Fédération internationale pour la recherche théâtrale (FIRT). Reprenant le débat des arts et des médias à sa base fonctionnaliste, Boenisch n’a aucune difficulté à démontrer que le théâtre remplit efficacement et depuis toujours sa fonction sociale et culturelle de média. ${ }^{27} \mathrm{Il}$ observe aussi que le théâtre se qualifie aisément comme « média de la représentation", qualité qu'il partage non seulement avec le cinéma et les jeux vidéo, mais avec les médias technologiques de transmission de l'information en temps réel.

Theatre also combines aspects of representational media, which foreground the creation of realities such as film and computer-games, with strategies of media aimed primarily at real-time presentation and communication, like the telephone, fax, and live broadcasting. For the creation of a reality that is happening in real time theatre is a hard medium to beat; for it makes worlds that are in a very tangible way real for the observers and they experience that world in actual time. (Boenisch 110)

Art de la représentation, donc de l'espace partagé et du temps réel, le théâtre est souvent défini et perçu comme un média fédérateur ou comme le média multimodal (Elleström) par excellence, 
qualité que peu d'autres médias partagent (l'opéra, les concerts de rock). Cette qualité, alliée à la fois au fait objectif qu'il préexistait à tous les médias de la modernité-les médias électriques et numériques-et à la dynamique de remédiation, explique l'importance de sa fonction et de son statut historiques. Il aurait servi et servirait encore (par exemple pour les jeux vidéos, le webfilm, la réalité virtuelle) d'incubateur et de matrice à la plupart des médias apparus depuis la révolution électrique dans le champ du divertissement. Or, si la remédiation marque aussi bien le « remédié » que le " remédiant » et si le jeu entre transparence et opacité ou effacement et affirmation identitaires du média est réel, l'histoire du théâtre pendant le Long Siècle (1880 à aujourd'hui) devrait porter les traces de ces moments cruciaux où le théâtre a été remédié (par un cryptomédia).

C'est ce principe, conforme à la fois aux théories de Bolter et Grusin, à celles des généalogistes et aux arguments clés de l'interartialité, qui sert de fondement à relecture de l'histoire du théâtre des cent dernières années que propose Peter Boenisch. Son hypothèse est la suivante : chaque fois que le théâtre a fait l'objet d'une remédiation, ses agents ont réagi en opacifiant sa médialité, pour ainsi mieux afficher sa singularité et affirmer sa nécessité et son identité. Boenisch brosse là, à grands traits, l'histoire intermédiale du théâtre depuis l'avènement de lélectricité, histoire qu'il articule en trois temps correspondant respectivement au triomphe du cinéma, à la percée de la télévision et de la vidéo, à la révolution numérique.

Le premier temps concerne donc le cinéma et se déroule durant le premier quart du $\mathrm{XX}^{\mathrm{e}}$ siècle. Cette époque, qui voit le triomphe des vues animées et qui annonce l'avènement du parlant, correspond à un changement radical : le mouvement de théâtralisation du théâtre- " theatricalism as anti-realism ", dirait Postlewait-qui rend visible aux spectateurs tout ce que, jusque-là, la scène à l'italienne sétait évertuée à leur cacher. Pour Boenisch, il sagit d'un repositionnement stratégique historique. Face à un rival dont les capacités illusionnistes-de reproduction du réel-sont réputées supérieures aux siennes, le théâtre réagit en affichant sa médialité (son dispositif de médiation). Ce mouvement, qui est incarné par certaines expérimentations des avant-gardes, par le constructivisme, puis, dans une certaine mesure, par les théâtres de Piscator et de Brecht, change le rapport traditionnel de la représentation au réel. Le passage métaphorique du miroir à la lampe par lequel Meyer Abrams illustre la nouveauté de l'art romantique pourrait s’appliquer à ce « théâtre 
théâtral » qui ne prétendrait plus être une pratique imitative mais, comme le rappelle Moser, « un art non-représentatif dont on rend compte non pas en termes de vérité et d’adéquation à un objet à représenter, mais en termes dénergie émotive " (Moser 74). On songe d’emblée à la biomécanique de Meyerhold. Ce phénomène correspond à ce que Bolter et Grusin rassemblent sous le terme d'hypermédiacie, dont ils relèvent justement l'effet paradoxal. L’immédiacie, provoquée par la transparence du média, résulte de l'illusion d'un accès immédiat-une coprésence-au réel (représenté). L'hypermédiacie peut, quant à elle, faire du média l'objet réel de sa propre représentation. Notons, par ailleurs, que c'est justement à cette époque de grande tension, où la place prépondérante du théâtre est menacée par l'envahissement du disque, de la radio et du cinéma, que sélabore un discours théâtral essentialiste fondé sur les concepts de présence, coprésence et live. Et c'est lépoque où Walter Benjamin développe son concept d'aura.

Le second temps relevé par Peter Boenisch porte sur la période 1950-1980 marquée par le triomphe de la télévision et l'apparition de la vidéo auxquels le théâtre réagit en s'affirmant comme " the residue of pure and authentic culture in a word of mass-media and television daftness "(Boenisch 103). Cette période est aussi celle de l'hégémonie du discours essentialiste qui oppose sans nuance le rapport "vrai ", intense, direct-on ne peut pas imaginer qu'il puisse être technologiquement médiatisé-d'un acteur communiant avec son public dans le plus grand dénuement. C'est le culte du partage de l'émotion inaltérée, dans un espace et un temps réels communs à l'acteur et au spectateur. La scène vide est la réponse du théâtre au bric-à-brac triomphant et tapageur des studios de télévision, de cinéma, d'enregistrement sonore et de radio où tout est artifice. Le vieux débat, qui oppose la nature à la technologie, connaît là un nouveau paroxysme. Aux accusations de trahison qui leur sont adressées, les technologies de reproduction sonore réagissent par le concept-slogan de " haute fidélité ». Mais rien n’y fait. Le théâtre célébré par Jerzy Grotowski et Peter Brook essaime, il domine la vague la plus turbulente (de 1968 à 1975) du mouvement de création collective. Le vide contre l'abondance, la vérité profonde contre le mirage du matérialisme. Le conflit n'est pas circonscrit à la scène des arts et des médias. Il embrase toute une société.

Le troisième temps se produit, toujours selon Boenisch, au cours des années 1990, alors que les technologies numériques envahissent les pratiques médiatiques et artistiques. Les fondements mêmes des concepts de présence, de coprésence, de direct, 
de représentation en temps réel, etc., sur lesquels le théâtre sétait basé pour défendre sa spécificité ontologique depuis plus d'un demi-siècle, sont ébranlés. L'avènement du numérique ouvre lère des migrations, des transferts, du recyclage, des contaminations et des fusions qui brouillent les catégories et pulvérisent les barrières identitaires. Ce qui, jusque-là, avait assuré la « différence » théâtrale sétiole inexorablement. Vigoureusement lancé par l'ouvrageclé de Philip Auslander, en 1999-qui, entre autres choses, questionne le concept cardinal de liveness que le théâtre sétait approprié-, le débat sur le discours essentialiste n’a perdu ni en vigueur ni en actualité comme en témoignent les rééditions subséquentes de cet essai majeur en 2002 et en 2008. Les changements vécus lors de ce troisième temps historique trouvent leur écho dans le champ heuristique avec léclosion de nouveaux concepts qui témoignent bien de l'effet du numérique sur la scène et dans les processus de création théâtrale : "effets de présence ", " effets de réel », « re-présentation ». Le terme même de théâtralité, abondamment utilisé au-delà de la scène (en politique, en design, dans les cours de justice, etc.), connait une perte de faveur dans le discours théâtral due, sans doute, à son lourd passé sémiologique. Il est progressivement supplanté par celui de performativité jugé plus apte à rendre compte des pratiques contemporaines.

Si les hypothèses de Peter Boenisch restent, à bien des égards, discutables, sa relecture de l'histoire théâtrale ne manque ni d'intérêt ni de justesse. Elle souligne bien l'intermédialité intrinsèque du théâtre qui, de temps immémoriaux, est une pratique du croisement, de l'emprunt, du recyclage. Il n'a eu de cesse de s'approprier les techniques, technologies, dispositifs ou stratégies d'autres médias, d'autres pratiques, d'autres institutions pour se renouveler et pour élargir son potentiel spectaculaire. Vu sous cet angle, il n'y a donc pas de différence ontologique entre l'adoption de la perspective, l'intégration de lélectricité, le recours au son reproduit ou l'ouverture aux technologies numériques. Le théâtre est le prototype même du média pique-assiette en perpétuelle transformation. Et c'est sur ce constat paradoxal du " always already new ${ }^{28}$ "(Gitelman) que se clôt la réflexion de Peter Boenish : la seule constance-d'aucuns diraient l'essence-du théâtre, ce qui le définirait ultimement, serait sa continuelle transformabilité ! Invoquant De Kerckhove, qui décrivait le théâtre « as a sort of prototype of imagination, a try-out space for new experiences, emotions, attitudes and reflexions » (De Kerckhove, 149), Boenisch conclut : 
I suggest that theatre turns into a new medium [souligné par l'auteur] whenever new media technologies become dominant, and, in addition, that the theatre adapts and disperses the new cognitive strategies, just as it did in ancient Greece. (Boenisch 111)

Ce sont justement ces incessants " twists and turns ", dont l'histoire théâtrale abonde, qui expliquent, selon Samuel Weber, la suspicion et la fascination dont le théâtre a toujours été l'objet :

Theater is a medium that, from Plato and Aristotle to the present, has been regarded with suspicion, fear and contempt-but also with fascination and desire-by a tradition seeking at all costs to keep the ground from slipping out from under its feet. The twists and turns of this medium, in its history as well as in its practice, are perhaps even more acute today, when the notion of " media " has become more ubiquitous and more elusive than ever before. (Weber 30)

Appartenant au groupe de chercheurs intermédiaux issus des études sur les nouveaux médias, Chiel Kattenbelt est l'auteur d'un autre article majeur pour l'intermédialité théâtrale, article largement inspiré de la pensée numérique. Les objets numériques, quelles que soient les combinaisons auxquelles on les soumet, ne perdent jamais leur intégrité à la différence des objets analogiques qui se transforment de génération en génération ou en se combinant à d'autres objets analogiques. L'influence de Lev Manovitch sur la pensée de Kattenbelt est manifeste et elle est totalement assumée :

The structure and language of the digital media, which we believe is so important to contemporary theatre and performance in the age of the intermedial is the clear focus of The Language of New Media. ${ }^{29}$ (Chapple et Kattenbelt 17)

Et la description que Manovitch fait des objets numériques et de leur traitement sert de fondement au modèle intermédial que développe Kattenbelt :

[a] new media object [affirme Manovitch] has the same modular structure throughout. Media elements, be they images, sounds, shapes, or behaviors, are represented as collections of discrete samples (pixels, polygons, voxels, characters, scripts). 
These elements are assembled into larger-scale objects but continue to maintain their separate identities. The objects themselves can be combined into even larger objects-again, without losing their independence. (Chapple et Kattenbelt 17)

Les caractéristiques du numérique-la préservation de l'intégrité des objets, leur remarquable capacité combinatoire et fusionnelle, leur indépendance réciproque, lomniprésence du vide (entre les signaux) - constituent le modèle fondamental de l'intermédialité et coïncident exactement avec la réalité théâtrale (telle quelle est vécue depuis la Grèce antique). Kattenbelt va encore plus loin que Boenisch : le théâtre n'est pas seulement intermédial, il est le site de l'intermédialité : « The first principle is that theatre is a hypermedium that incorporates all arts and media and is the stage of intermediality » (Chapple et Kattenbelt 20).

Le recours au terme "hypermedium» crée évidemment un flottement puisqu'il renvoie d'emblée aux concepts d’opacité et d'hypermédiacie de Bolter et Grusin. Il est clair cependant que, dans l'esprit de Kattenbelt, l'opacité théâtrale n'est pas une opacité comme les autres. Il faut donc prendre le terme hypermedium en son sens littéral de média fédérateur, de "média des médias", " home to all ", foyer, site, lieu de convergence et de rencontre " where the art forms of theatre, opera and dance meet, interact and integrate with the media of cinema, television, video and the new technologies » (Chapple et Katttenbelt 24). Cette hospitalité se reflète dans le traitement singulier que le théâtre réserve aux autres objets médiatiques, aux technologies, aux autres médias qui entrent dans le processus de la création ou dans celui de la représentation théâtrale :

[F]ilm, television and digital video are technology-based media that can record and play back everything that is visible and audible, within their specific ranges of sensitivity, but they cannot incorporate other media without transforming them under the conditions of the specificity of their own mediality. At the very most, media can remediate (Bolter and Grusin 1999) other media, which implies in the end a refashioning. Clearly, theatre is not a medium in the way that film, television and digital video are media. However, although theatre cannot record in the same way as the other media, just as it can incorporate the other arts, so it can incorporate all media into its performance space. It is in this capacity that I regard theatre as a hypermedium. (Kattenbelt 37) 
Kattenbelt n'utilise pas le terme d'interartialité, sans doute parce qu'il considère inutile de distinguer à ce stade de sa réflexion les arts des autres médias. Mais il observe clairement que le phénomène d'opacification résultant de cette action fédératrice du théâtre est atypique. Le théâtre, affirme-t-il, est « the only art capable of incorporating all other arts without being dependent on one of these in order to be theatre " (Kattenbelt 32). La formule pourrait être inversée-les autres arts conservent leur intégrité et leur indépendance-et elle pourrait être élargie à l'ensemble des médias « remédiés » par la théâtralité.

Pour rendre compte de cette remédiation fédératrice du théâtre-toute fédération devant, en principe, respecter l'identité et l'autonomie relative de ses composantes-, il paraît plus juste de qualifier le théâtre de métamédia. Et l'opacité particulière du théâtre serait une forme de métamédiacie dont Kattenbelt décrit ainsi la logique active :

Theatre provides film, television and digital video a stage, that is to say a performative situation [...]. [T] he other media are not just recordings on their own, but at the same time and above all theatrical signs. Operative as part of theatre, the other media become signs of signs as opposed to signs of objects. (Kattenbelt 37)

On aurait souhaité que Kattenbelt explicite davantage la mécanique de cette dualité. Retenons-en ceci : tout en préservant-et en jouant de-lopacité de ses composantes, la théâtralité leur confère une plus-value spectaculaire qui, en même temps, les solidarise et conforte l'usager-spectateur et l'usager-acteur dans cette conviction simple : ils sont au théâtre, non à un concert rock, non à un film, non à un procès en cour.

\section{La sociomédialité et la " théorie des restes": étude de cas sur la résistance médiatique}

Moser, Boenisch et Kattenbelt contribuent, chacun à sa façon, à lédification de l'intermédialité théâtrale. Rappelons brièvement leur apport respectif. Moser précise les modalités de la remédiation dont il démontre la subtilité et la diversité. On savait que la remédiation avait des effets en aval et amont-sur le remédiant comme sur le remédié-, mais Moser montre clairement que, dans cette dynamique complexe, les présupposés habituels ne tiennent pas. Le média dominant n’est pas nécessairement celui 
qui impose son opacité et le média intégrateur peut s'effacer ou sopacifier selon les enjeux de la création. Car, rappelle Moser, un art a d'abord pour fonction de créer des œuvres et ses stratégies changent d'une œuvre à l'autre.

Boenisch fait un cheminement inverse à celui d'Auslander et des généalogistes. Il revisite les grandes mutations médiatiquesde l'électricité au numérique-du point de vue, non pas des médias émergés de la série culturelle " théâtre », mais du théâtre lui-même; non pas du remédiant triomphant que décrivent les généalogistes, mais du remédié qui tente de survivre. Quant aux rapports de causalité qu'il établit entre ces moments d'émergence et les ruptures et innovations qui marquent le théâtre du Long Siècle, ils ont le mérite de rappeler que la percée de nouveaux médias majeurs relève toujours de vastes mouvements d’ensemble qui touchent toutes les sphères de la société et des savoirs.

La perspective de Kattenbelt est différente. Délaissant le macro pour le micro, il observe méticuleusement le fonctionnement interne du théâtre à partir du modèle du numérique. Ses conclusions confirment cependant les précédentes : le théâtre n'est ni un art ni un média comme les autres, c'est un hypermédia.

On l'aura constaté, le grand absent de toutes ces théories est l'usager. Moser l'ignore totalement; quant à Boenisch et Kattenbelt, s'ils soulignent bien l'importance de l'interaction scène-salle, de la co-construction du sens du spectacle, de la coprésence, ils s'en tiennent, en ce domaine, à de vagues généralités. L'usager est évoqué, il n’est pas convoqué. Or, il n’y a ni essentialisme ni déterminisme dans la conjoncture médiatique. Les médias, insistent Bolter et Grusin, ne peuvent être saisis isolément les uns des autres. J'ajouterais qu'ils ne peuvent pas davantage lêtre en faisant abstraction du milieu où ils opèrent, d'où ils émergent, où ils se transforment et disparaissent. Et c'est justement là l'objet de la sociomédialité : l'étude dynamique ${ }^{30}$ des socialités constitutives des médias.

Si l'effet des médias sur la société a déjà fait l'objet de vastes travaux dans des champs aussi diversifiés que la sociologie, l'anthropologie, les sciences cognitives, léconomie, la médiologie ou la philosophie, on sait encore peu de choses des mécanismes complexes, rhizomiques, qui lient le devenir du milieu et du média. Sous quelles pressions, en vertu de quelles actions, de quelles normes, de quels hasards ou accidents un média émerge$\mathrm{t}$-il, se transforme-t-il, disparaît-il ? Les réponses à ces questions résident moins dans l'examen des réussites exemplaires-le cinéma, le disque, la radio, la télévision-que dans les tentatives 
inabouties, les rejets, les processus suspendus ou les échecs. Bolter, Grusin et les généalogistes nont pas eu besoin d'intégrer les usagers autrement que comme une masse informe d'agents anonymes et interchangeables dans les modélisations qu'ils ont tirées de la genèse des grands médias contemporains. À la théorie des grands modèles, il faut donc ajouter la théorie des restes, innombrables, où l'action du milieu est plus palpable si l'on veut mieux comprendre le rôle de ce dernier. Pour l'illustrer, jévoquerai, en guise de conclusion, l'histoire tourmentée de la lente intégration du son médiatisé aux scènes et salles de théâtre. Délaissant le champ du numérique actuel, remontons à lépoque de lélectricité triomphante.

Alors que le théâtre occidental intègre rapidement les innovations apportées par l'électricité en matière déclairage, il attend près de trois quarts de siècle (les années 1950) avant de permettre au son électrique de se déployer sur ses scènes, dans ses salles et dans ses processus créatifs. Il a donc fallu à peine dix ans à la lampe à incandescence d'Edison (inventée en 1879) pour chasser léclairage aux becs de gaz de l'immense majorité des scènes et salles de théâtre d'Occident, mais trois quarts de siècle après l'invention du téléphone (Bell, 1876), du microphone (Berliner, 1877 ; Edward Hugues, 1878) et du phonographe (Edison, 1877), le son médiatisé ${ }^{31}$ demeurait une rareté sur l'immense majorité des scènes théâtrales. Pendant ce temps, le cabaret (chanson, humour-1936), la radio (1920) et le cinéma parlant (à partir de 1927) prenaient, grâce à lui, l'essor phénoménal qu’on sait ! Le son médiatisé a donc donné naissance à trois médias majeurs en moins d'un demi-siècle-le phonogramme-disque, la radio et le cinéma parlant—qui ont affaibli le statut du théâtre dans le champ en pleine expansion du divertissement qu'il dominait jusque-là.

Si des questions de sécurité ont indéniablement facilité et accéléré le passage de léclairage au gaz à léclairage électrique, cela justifie-t-il un décalage de soixante ans ? Sûrement pas ! Lapparente incapacité du son médiatisé à pénétrer les scènes théâtrales de 1877 au milieu des années 1950-date où on commence à "sonoriser " les scènes et à utiliser des bandes$\operatorname{sons}^{32}$-relève d'un phénomène de résistance médiatique qui a pour effet d'exclure, éviter, retarder l'introduction dans un média donné d'une nouvelle technologie, d'un nouvel objet médiatique ou d'un nouveau média. La résistance suspend toute possibilité de remédiation de l'intrus en question. Quelle en est la cause?

Un premier réflexe serait évidemment, dans le cas qui nous occupe, de l'imputer aux insuffisances de la technologie. Mais 
comment expliquer que ces « insuffisances » auraient maintenu le son médiatisé au seuil des théâtres tout en assurant son succès phénoménal à l'extérieur de la scène : sur disque, à la radio et dans les salles du cinéma ? La question des insuffisances est complexe, elle réfère à un « horizon d'attente auditive " difficile à reconstituer, avec tout ce que cette attente suppose de déterminisme idéologique, de présupposés esthétiques et de conditionnement physique. On ne peut pas écarter l'hypothèse des "insuffisances ", mais elle ne peut pas tout expliquer, surtout sur une période de soixante-quinze ans riche en percées technologiques très variées dans le domaine. La cause profonde de toute résistance médiatique est nécessairement sociomédiale, elle relève du milieu. C’est la résistance du milieu à tout changement qui aurait pour effet de-trop-transformer son usage du média ou de déprécier sa propre perception du média. Si ce concept de résistance s’applique à tous les types de médias, il est particulièrement actif dans les médias artistiques.

On peut supposer que le son médiatisé n’a pu pénétrer le théâtre-dans le lieu et dans ses processus-qu'à partir du moment où il a cessé dêtre perçu comme une menace à ses fondements, c'est-à-dire à sa théâtralité. Mais paradoxalement, cette fermeture a peut-être été encore plus préjudiciable au théâtre en favorisant le déploiement de médias sonores à grand succès qui l'ont ensuite concurrencé dans le champ du divertissement et qui ont mis fin à son statut de pratique majoritaire. Pire encore, ces médias ont débauché son public, ses agents et se sont parfois même approprié son organisation institutionnelle, ses modalités (la frontalité, l'expérience commune), ses espaces (dans le cas du cinéma), pillant sans retenue la série culturelle " théâtre ». La résistance du théâtre au son médiatisé reste conjoncturelle, dans le temps, bien sûr-puisque le son médiatisé sera finalement accepté et totalement "naturalisé » par la scène-, mais aussi dans l'espace. En effet, le théâtre n’a pas hésité à utiliser le son électrique hors de son enceinte pour son rayonnement, grâce au théâtrophone, ${ }^{33}$ au théâtre radiophonique et au disque qui étaient le fait des artistes de théâtre et qui diffusaient des produits sonores issus de la scène. En d'autres termes, le son médiatisé qui est tout à fait acceptable hors scène pour des artistes-et évidemment pour les spectateurs-de théâtre, se révèle inacceptable sur scène, autrement quà titre de curiosité. Et il n'est pas anodin que les premières percées du son médiatisé au théâtre se soient effectuées progressivement dans les formes théâtrales les plus récentes, les moins structurées et les moins prestigieuses-la revue, les variétés, la 
féérie et les varieties, le vaudeville américain, le Grand-Guignol, le burlesque.

La résistance médiatique est clairement liée à la scène traditionnelle, à son fonctionnement et à son statut. C'est elle que le son médiatisé menace en tant qu'espace consécrateur, en tant que lieu de la performance, en tant que site historique du corps agissant et de sa voix. Jusquà l'avènement du son médiatisé, rappelons-le, la scène avait été, de mémoire de spectateur et d'acteur, l'unique lieu de consécration des artistes. Le studio de son, comme lieu concurrent de profération de la voix (le disque, la radio) portait atteinte à ce statut unique que Giusy Pisano qualifie de "sacré " justement en raison du pouvoir consécrateur qui lui était attaché. La scène était le lieu de cette performance toujours renouvelée du corps sonore qui l'habite et qui lui confère ses qualités auratiques. Avant le son médiatisé, une "belle voix » était nécessairement une " forte voix » et la scène était le lieu de cette performance vocale « forte » et directement partagée. Mais que devient la performance quand la machine intervient entre l'acteur et le spectateur et qu'advient-il de la scène?

L'insistance avec laquelle l'industrie de reproduction du son proclame, ainsi que le rappelle Jonathan Sterne, la « fidélité » puis la « haute fidélité » de ses appareils-sans qu’on sache très bien à quoi ils étaient fidèles ! - n’avait pas d’autre but que de contrer un sentiment de "trahison " largement répandu chez les artistes et les spectateurs (Sterne 215-225). L'intrusion du son médiatisé sur scène était présentée sous l'angle d'une double agression : d'abord, celle de la "nouvelle culture décadente " des amusements mécaniques contre l'" autre » culture, avec ce que cela induit de nostalgie-la disparition de l'aura-; ensuite celle d'une atteinte à la «présence » de l'acteur si liée à l'épistémè théâtrale. Le son médiatisé, en instaurant une première rupture historique de la voix et du corps (Dolar 9-12), brisait l'unicité de cette présence millénaire et répandait la pratique de l'écoute acousmatique qui banalisait la séparation du son et de sa source, de la voix et du corps.

Il est difficilement concevable que les artistes et le public aient accepté, sans résister, que leur scène soit ainsi dépossédée de son pouvoir consécrateur historique, privée de l'aura qui faisait son prestige et son attrait, qu'elle devienne le site d'une performance " inauthentique " et qu'ils s'y contentent d'une présence fragmentée. Pourquoi alors, quelques décennies plus tard, le son médiatisé entrait sur scène et dans le processus de création théâtrale sans provoquer de réaction, sans susciter de débat ? L'étude reste à faire, mais une chose est indéniable : la configuration des 
médias avait changé, lécoute acousmatique ${ }^{34}$ avait été naturalisée. De toute évidence, cétait une autre sociomédialité !

\section{Conclusion}

Comme ce dernier exemple l'indique encore, une pratique médiatique, qu'elle soit artistique ou non, met en jeu des personnestous les types d'usagers-, des valeurs, des objets, des techniques, des savoirs qu'on peut difficilement isoler et dont la conjoncture est elle-même perpétuellement changeante, toujours croisée, toujours nouvelle et en même temps prodromique d'une nouveauté à venir... qui puise dans le passé !

On ne saurait trouver illustration plus convaincante de cette réalité intermédiale que le théâtre lui-même ! Sa transformation continuelle, alimentée par les attentes et les besoins sans cesse redéfinis de ses multiples agents, par les avancées-ou ce qui est perçu tel-d'autres pratiques et de la technique, par les changements du cadre légal, économique, etc., présente peu de signes de régularité et demeure largement imprévisible. Au vertige que produit ce mouvement dynamique pour quiconque l'observe, s'ajoute la perte des références coutumières : les barrières disciplinaires seffondrent, la langue n'a plus son statut de médiateur universel indiscuté, l'hybridation se généralise, favorisée par des technologies plus souples, plus puissantes-mais plus complexes. Et il est désormais clair, comme le démontrent les rapports complexes liant le théâtre au cinéma, à la radio et à la télévision, par exemple, que le cannibalisme intermédial-entre médias(ce qui inclut le cannibalisme interartial) est un élément fondamental de la genèse et de la dynamique des médias qui émergent du milieu qu'ils contribuent à transformer.

Il nest pas étonnant, dans ce contexte de grande instabilité, que des résistances se manifestent qui peuvent prendre la forme de résistance médiatique, au sens où nous venons de le voir, ou de resserrement autour d'une identité qui préserverait l'essentiel, celui-ci-le resserrement-allant souvent de pair avec celle-là-la résistance médiatique. Il n'est pas étonnant non plus que de nouvelles approches, comme l'intermédialité, soient développées dans ces circonstances, non pas dans le but chimérique de modéliser cette réalité fuyante et, pour tout dire, insaisissable dans sa nature et dans sa conjoncture, mais pour en doser, justement, l'infini. 


\section{NoTES}

1 Relèvent de cette matérialité les matériaux constitutifs (lieu, corps, voix, image, son, etc.), les supports matériels (pellicule, ruban magnétique, plateau, scène, corps encore-comme « site d'inscription » -, etc.), les objets techniques et technologiques appartenant au dispositif médiatique.

2 En dépit des travaux en épistémologie de l'intermédialité, nous n’en sommes pas encore à une définition stable. Celle que j'adopte découle des travaux du Centre de recherche sur l'intermédialité de l'Université de Montréal.

3 J'emploie ici « avènement » dans le sens proposé par André Gaudreault et Philippe Marion pour définir la naissance « finale » du média qui correspond à son autonomisation et à son institutionnalisation (Gaudreault et Marion 20-36).

4 C'est le sous-titre de son article.

5 Cette définition provient, pour l'essentiel, des travaux du Centre de recherche sur l'ntermédialité (CRI) de l'Université de Montréal. Elle fait écho à d'autres entreprises définitoires menées séparément et ailleurs, dont celles de l'École allemande de l'intermédialité (particulièrement les définitions de Christopher Balme et Jens Schröter). Pour un rapide survol du sujet, voir l'article de Freda Chapple et Chiel Kattenbelt, « Key Issues in Intermediality ».

6 Ce que nous nommons « sociomédialité ».

7 D’ailleurs, le Centre de recherche sur l'intermédialité de l'Université de Montréal est historiquement plus lié à la révolution électrique (fin $\mathrm{XIX}^{\mathrm{e}}$ et début $\mathrm{XX}^{\mathrm{e}}$ ) quà la révolution numérique survenue cent ans plus tard.

8 Dès 1988, Carolyn Marvin publiait un ouvrage-clé sur la question, soulignant, elle aussi, tous ces phénomènes de transfert et l'importance du milieu dans la genèse des médias électriques (When Old Technologies Were New-Thinking About Electric Communication in the Late Nineteenth Century). Les ouvrages subséquents de Jonathan Sterne (The Audible Past, 2003), de Siegfried Zielinski (Deep Time of the Media, 2002) et de Lisa Gitelman (Always Already New, 2006) vont dans le même sens.

9 Qu'on songe au passage des premiers baladeurs analogiques de type Walkman aux micro-lecteurs portatifs MP3, du téléphone fixe au téléphone cellulaire et à la dernière génération de I Phone.

10 D’abord la vidéo, ensuite l'ordinateur puis les technologies numériques.

11 Et on peut comprendre pourquoi. Comment, en effet, se spécialiser hors d'une discipline? Voir, à propos de cette crise l'article percu- 
tant de James Cisneros, « Remains To Be Seen-Intermediality, Ekphrasis, and Institution ».

12 Les créations des Aveugles de Maeterlinck (en 2002) et d'Une fête pour Boris de Thomas Bernhard (en 2009) par Denis Marleau, fondées sur la projection de vidéos sur des masques représentant des visages, entrent dans cette catégorie de spectacles hautement technologiques.

13 Pour une synthèse de ce vaste mouvement collectif à léchelle internationale, voir Jane Baldwin, Jean-Marc Larrue et Christiane Page, Vies et morts de la création collective-Lives and Deaths of Collective Creation.

14 Pour W.J.T. Mitchell, le tournant optique ne relève pas seulement $\mathrm{du}$ fait que les images qui nous environnent transforment notre monde mais que, de plus en plus, elles le forment. Les images jouent ainsi un rôle sans cesse grandissant dans la construction de notre réalité sociale. Ceci renvoie à cette autre litéracie qu'est la litéracie de la différence évoquée par Mariniello (voir W.J. Thomas Mitchell: «The Pictorial Turn» dans Picture Theory. Essays on Verbal and Visual Representation).

15 Voir l'ouvrage de Jean-Louis Déotte, Lépoque des appareils.

16 Lyotard identifie ainsi trois époques de la surface d'inscription, dont la première est la narration.

17 Mais la situation tend à changer, signe de l'avènement de cette nouvelle litéracie de la différence. La question du son au théâtre, largement ignorée des historiens et peu abordée par les théoriciens du théâtre, suscite depuis quelques années un véritable engouement (comme en témoigne le développement des sound studies aux Etats-Unis) qui accorde une place majeure, égale à celle de l'image, à la dimension sonore du théâtre-le son de la scène comme le son de la salle. L’Équipe internationale sur le son fondée, en 2007, par le Centre de recherche sur l'intermédialité (CRI, Montréal) et le Laboratoire ARIAS du Centre national de recherche scientifique (CNRS-ARIAS, Paris), a justement pour objectif de proposer une première histoire du son au théâtre.

18 Lars Elleström identifie quatre «modalités » de médiation nécessairement présentes dans tout média, qu'il soit artistique ou non : modalité matérielle, sensorielle, spatiotemporelle, sémiotique (pour plus de détails, voir Lars Elleström, « The Modalities of media-A Model for Understanding Intermedial relations »).

19 Louvrage n’a pas encore été traduit en français et est peu connu en France.

20 Lindustrie du disque-donc de l'enregistré—a fait de même avec les enregistrements dits live. 
21 Bolter et Grusin démontrent que le média qui fait l'objet d'une remédiation-et qui survit-y réagit en se transformant. Ainsi, la remédiation affecte aussi bien le média remédiant que le média remédié, ce dont l'histoire du théâtre fournit d'innombrables exemples.

22 Moser voit plutôt ces phénomènes dans une perspective artistique considérant l'intermédialité comme une archéologie de l'interartialité.

23 Ce qui est l'une des caractéristiques de tout un champ de l'art moderne depuis Marcel Duchamp, mais aussi de l'hypermédiacie telle que la définissent Bolter et Grusin (quand elle devient autoréférentielle).

24 Voir « Art, Practice, and Narrative » (1988), « Identifying Art » (1993), « Historical Narratives and the Philosophy of Art " (1993), tous repris dans : Noël Carroll, Beyond Aesthetics. Philosophical Essays. Même si son approche de l'art n'est pas à proprement parler définitionnelle, Carroll considère, tout comme Levinson, que l'historicité est une caractéristique déterminante de l'art. Le fait d'être une pratique intrinsèquement liée à une tradition est une propriété essentielle de l'art.

25 Ainsi Moser montre comment dans le film Caravaggio de Derek Jarman, la médialité du film s'efface au profit de celle de la peinture, alors que dans le film Passion de Jean-Luc Godard, c'est l'inverse.

26 On peut cependant présumer qu'il s'applique à la plupart des arts du spectacle vivant.

27 « Processing, transmission, and storing of information ». Ces processus, définis par Friedrich Kittler dans Discourse Networks 1800/1900, se différencient peu de la définition fonctionnaliste de Frédéric Barbier et Catherine Lavenir.

28 C'est ainsi que Lisa Gitelman caractérise la nouveauté présumée des médias qui n'en est pas une. C'est même le titre de son ouvrage: Always Already New-Media, History and the Data of Culture.

29 C'est le titre de l'ouvrage-clé que Manovitch publie en 2001 (voir Freda Chapple et Chiel Kattenbelt. « Key Issues in Intermediality »).

30 Cette dynamique est nécessaire puisque ces socialités ne cessent de changer.

31 Par « son médiatisé », il faut entendre tout son conservé, communiqué ou réactualisé technologiquement. Cela inclut (1) le son acoustique produit par cornet acoustique et saisi-puis lu-sur des feuilles d'étain (fixées sur des rouleaux), sur des rouleaux de cire ou des disques, et (2) le son électrique produit par micro et reproduit par haut-parleur. Ces deux types de sons médiatisés se sont déve- 
loppés concurremment. Le son électrique a supplanté le son acoustique à partir de 1925 .

32 L'un des premiers utilisateurs d'une bande-son au théâtre est le cinéaste Luchino Visconti. On peut dire que le théâtre a remédié le cinéma pour cet aspect, du moins.

33 Système permettant à un auditeur se trouvant dans un café situé à l'extérieur du théâtre découter le spectacle donné sur une scène (grâce à un micro sur scène).

34 R. Murray Schafer a créé le terme « schizophonie " (schizophonia) pour exprimer cette séparation du son original et de sa reproduction électroacoustique (Schafer 131).

\section{OUVRages CITÉS}

Abrams, Meyer H. Abrams. The Mirror and the Lamp: Romantic Theory and the Critical Tradition. New York: Norton, 1953.

Auslander, Philip. Liveness: Performance in a Mediatized Culture. 1999. London and New York: Routledge, 2008.

- Presence and Resistance-Postmodernism and Cultural Politics in Contemporary American Performance. 1992. Ann Harbor: U of Michigan P, 1994.

Baldwin, Jane, Jean-Marc Larrue et Christiane Page (sous la dir. de). Vies et morts de la création collective-Lives and Deaths of Collective Creation. Boston : Vox Theatri, 2008.

Barbier, Frédéric et Catherine Bertho Lavenir. Histoire des médias : de Diderot à Internet. Paris : Armand Colin/ Masson, 1996.

Blakesley, David. «A Review of Remediation: Understanding New Media. » Kairos 6.1 (2001) : np. Web. 19 janvier 2009.

Boenisch, Peter. « Aesthetic art to aisthetic act : theatre, media, intermediality ». Chapple et Kattenbelt 103-116.

Bolter, Jay David et Richard Grusin. Remediation-Understanding New Media. Cambridge: MIT P, 2000.

Carroll, Noël. Beyond Aesthetics. Philosophical Essays. Cambridge: Cambridge UP, 2001.

Chapple Freda, et Chiel Kattenbelt (sous la dir. de). Intermediality in Theatre and Performance. Amsterdam and New York: Rodopi, 2006.

— , et Chiel Kattenbelt, « Key Issues in Intermediality ». Chapple et Kattenbelt 11-25.

Cisneros, James. « Remains To Be Seen-Intermediality, Ekphrasis, and Institution ». Froger et Müller 15-28.

Davis, Tracy C. et Thomas Postlewait. Theatricality. Cambridge :

Cambridge UP, 2003. 
De Kerkhove, Derrick. « Theatre as Information Processing, " Western Culture in Modern Drama 25.1 (1982): 143-153.

Déotte, Jean-Louis, Marion Froger et Silvestra Mariniello (sous la dir. de). Appareil et intermédialité. Paris : L’Harmattan, 2007.

—. Lépoque des appareils. Paris : Éditions Lignes \& Manifestes, 2004.

—. «Révolution des appareils (notes pour un programme de recherche) ». L'Art au temps des appareils. Pierre-Damien Huyghe (sous la dir. de). Paris : L'Harmattan, 2005. 261-278.

Dolar, Mladen. A Voice and Nothing More. Cambridge and London: MIT Press, 2006.

Elleström, Lars. « The Modalities of Media-A Model for Understanding Intermedial Relations ». Media Borders, Multimodality and Intermediality. New York: Palgrave Macmillan, 2010. 11-50.

Froger, Marion. « Appareil et intermédialité-Introduction ». Appareil et intermédialité. Déotte, Froger et Mariniello 5-16.

—, et Jürgen E. Müller (sous la dir. de). Intermédialité et socialitéHistoire et géographie d'un concept. Münster : Nodus Publikationen, 2007.

Garneau, Michèle. «Cinéma, communauté, appareil : réflexion à partir de Close-up d'Abbas Kiarostami et Salaam Cinema de Moshen Makhmalbaf ». Déotte, Froger et Mariniello 75-96.

Gaudreault, André et Philippe Marion. " Un média naît toujours deux fois » Sociétés et représentations. Numéro spécial sur «La Croisée des médias " 9 (2000) : 21-36.

Gitelman, Lisa. Always Already New-Media, History and the Data of Culture. Cambridge: MIT P, 2006.

Johnson, Barbara. The Critical Difference. Baltimore: John Hopkins UP, 1980.

Kattenbelt, Chiel. «Theatre as the art of the performer and the stage of intermediality ». Chapple et Kattenbelt 29-40.

Kittler, Friedrich. Discourse Networks 1800/1900 (trad. de M. Meeter). Stanford: Stanford UP, 1992.

Mariniello, Silvestra. « La litéracie de la différence ». Déotte, Froger et Mariniello 163-188.

Marvin, Carolyn. When Old Technologies Were New-Thinking About Electric Communication in the Late Nineteenth Century. New York: Oxford UP, 1988.

Méchoulan, Éric. «Intermédialités : Le temps des illusions perdues ». Intermédialités. Numéro special sur « Naître » 1 (2003) : 9-28.

Mitchell, W.J. Thomas. «The Pictorial Turn ». Picture Theory. Essays on Verbal and Visual Representation. Chicago: U of Chicago P, 1994. 11-35. 
Moser, Walter. « L'interartialité : pour une archéologie de l'intermédialité ». Froger et Müller 69-92.

Müller, Jürgen E. «Séries culturelles audiovisuelles ou : Des premiers pas intermédiatiques dans les nuages de l'archéologie des médias ». Froger et Müller 93-110.

Sauter, Willmar. The Theatrical Event: Dynamics of Performance and Perception. Iowa City: U of Iowa P, 2000.

Schafer, R. Murray. Le Paysage sonore. Paris : JC Lattès, 1991.

Sterne, Jonathan. The Audible Past-Cultural Origins of Sound Production. Durham \& London: Duke UP, 2003.

Truax, Barry. Acoustic Communication. Norwood: Ablex, 1984.

Véron, Eliseo. « De l'image sémiologique aux discursivités ». Hermès. 13-14 (1994) : 45-64.

Villeneuve, Johanne. «Loralité, l'intermédialité et la question de l'appareil de la technique et des enchantements ». Déotte, Froger et Mariniello 207-222.

Weber, Samuel. Theatricality as Medium. New York: Fordham UP, 2004. 